\title{
Optimal Bidding Strategy for Profit Maximization of Generation Companies under Step-Wise Bidding Protocol
}

\author{
Ajay Bhardwaj $^{\# 1}$, Akash Saxena ${ }^{\# 2}$, Tanuj Manglani ${ }^{\# 3}$ \\ ${ }^{\# 1}$ M. Tech. Scholar, Yagyavalkya Institute of Technology, Jaipur \\ 1ajayb23@gmail.com \\ ${ }^{\# 2}$ Professor, Swami Keshvanand Institute of Technology, M\&G, Jaipur \\ 2aakash.saxena@hotmail.com

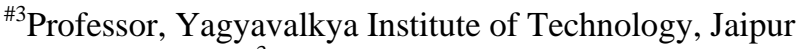 \\ 3idtanuj@gmail.com
}

\begin{abstract}
Deregulation of electricity industry has completely transformed the present power system. The generation companies (Gencos) are now interested in maximizing their profit by optimally strategizing their bids. In an oligopolistic market environment, only a few independent power suppliers can increase their own profit through strategic bidding. The profit depends to a great extent on imperfect knowledge of rival companies.

In this work, a new approach is developed for power suppliers to bid optimally for enhancement in their profits. The probability density function is used to determine the rival's behavior. An optimization routine has been established and Grey Wolf Optimizer (GWO) is used to find the optimal solution to the bidding problem. The approach is validated on four generators test system. The results are also compared with the application of Genetic Algorithm (GA), Particle Swarm Optimization (PSO) and Biogeography Based Optimization (BBO) algorithms. Comparison of the results shows that GWO is an effective solution provider.
\end{abstract}

Keywords - Electricity Market, Bidding Strategy, Monte Carlo Simulation, Grey Wolf Optimizer

\section{INTRODUCTION}

The electricity supply industry is going through a revolutionary age. The whole power system has been restructured and electricity has now become a commodity. With this, the term market is now associated with the power system. Despite this huge transformation, the electricity markets are still not perfectly competitive and only a few generation companies can participate in it.

In this oligopolistic market environment, there is limited no. of buyers and sellers due to large investment size and transmission constraints [1]. Each participant wishes to maximize their own profits. They submit their bid in the sealed envelope and submit it to market operator. The market operator decides the bid to be selected. The suppliers may bid at a price higher than their production cost to increase their profits but they have the risk of losing the competition. In pay as bid auction market with uniform market clearing price mechanism, a higher bid means higher profit. Therefore, it is a complex problem for generation companies to decide the optimal bid.

Various researchers have done considerable work in recent years on this problem. Optimal bidding strategy was developed among competitive generators in the imperfect environment of electricity markets in [1]. A literature survey has been presented for strategic bidding in competitive electricity markets in [2]. Dynamic programming approach was used to formulate the bidding problem in [3]. E. S. Huge et al. proposed heuristic approach based method to solve optimal bidding strategy in [4]. Li Ma et al. in [5] proposed Monte Carlo approach to solve optimal bidding problem. The normal probability distribution function was used to describe the rival's behavior and GA was applied to find an optimal bid. Multistage probabilistic bidding decision problem was addressed in [6]. Markov decision process was applied to solve this problem. In a day-ahead energy market, the strategic bidding was developed using GA in [7]. The same methodology was adopted to determine the bidding strategy for spinning reserve market in [8]. Intertemporal operating constraints were not included in these works and market model failed to represent the real market dynamic conditions. In [9], C.W. Richter et al. proposed bidding strategies using Genetic Programming (GP) with finite state automata. The GP-Automata resulted in successful bidding strategy. The information regarding unit commitment schedules and forecasted prices were not taken into account in this work. P. Bajpai et al. proposed fuzzy adaptive particle swarm optimization to formulate bidding strategy in uniform price spot market in [10]. R. W. Ferrero et al. presented game theory based approach to simulate decision making regarding offered prices in a deregulated market environment in [11]. In [12], Karl seeley et al. analyzed electricity market rules and their effects on strategic behavior. Their work showed that it was even possible for even mid-price suppliers to create congestion problems through gaming in a non-congestive system. S. Hao [13] proposed optimal bidding strategy 
of suppliers by solving a set of differential equations that specify the necessary conditions for bidders to maximize their expected payoffs. Ramping limits were ignored in this work. The explicit analysis of gaming and price spikes in the electricity market was presented in [14]. The author formulated a prisoner's dilemma matrix game and introduced the notion of opportunistic tacit collusion to explain strategic bidding behavior in which suppliers withhold generation capacity from the market to drive up prices. Wang Xian et al. [15] presented a mixed nonlinear complementarity problem by combining the Karush-Kuhn-Tucker conditions of all strategic generating companies. The results of this work showed that the generating companies can exercise their market power by over production under congestion. Binary expansion approach to solve optimal bidding problem was presented in [16]. The author solved the problem of strategic bidding under uncertainty in short-term electricity markets. In [17], author proposed a methodology to determine the optimal bidding strategy of a retailer in the short term electricity markets. GA was used to optimize the parameters defining the best purchasing strategy. Mostafa Kazemi et al. [18] presented a combined scheduling and bidding algorithm for constructing the bidding curve of an electric utility that participated in the day-ahead energy markets. The uncertainty involved regarding electricity markets was modeled using information-gap decision theory. S. Jalal et al. [19] proposed a mathematical model for large consumers to alter pool prices to gain more benefit in deriving bidding strategies. Uncertainty was modeled by a stochastic complimentarity model. Modeling of Risk management was not done in this work. In [20], a risk constrained bidding model for generation companies competing in a poolbased electricity market was presented. The dynamic programming algorithm was used to formulate strategic bidding problem. The bidding model took into account the uncertainty in system demand. The risk management was also integrated into the bidding model. Fco. Alberto Campos et al. [21] proposed a novel methodology to obtain real bidding curve for the spinning reserve market. The reserve cost curve was constructed by considering the day ahead market opportunity cost. This methodology was used in one of the prime power producers of Spain. BBO approach was used to maximize profit of generation companies in [22]. In [22], a generation company maximized its own profit while also maximizing profit of rival companies. The method predicted bid prices of rivals stochastically and then generated bid prices of the generation company whose profit was maximized.

Given this background, the work presented in this paper aims at maximizing the profit of a generating company while taking social welfare into account. The GWO [23] is used to secure the purpose to obtain bidding strategy for maximizing the profit of Genco. GWO is a new Meta heuristic algorithm inspired by grey wolves. It mimics the leadership hierarchy and hunting mechanism of grey wolves in nature. The algorithm provides very competitive results compared to other well known Meta heuristics algorithms. A comparison is also has been done between GWO and other heuristic algorithms (GA, PSO, BBO) in this paper. The remaining part of the paper is presented as follows; in section II and III, problem description and proposed solution algorithm along is presented. In section IV, simulation results are presented and finally, in section V, the conclusion and future scope of the work are presented.

\section{PROBLEM DESCRIPTION}

Consider a system consists of independent $\mathrm{N}+1$ generators. To maximize the profit, an optimal bidding strategy has to be developed for generator A, with $\mathrm{N}$ rivals in the market. Suppose that each generator has only one registered unit. In the day-ahead market, each generator bids for every one hour trading period under step wise block bidding protocol, uniform market clearing price (MCP) and sealed auction system, using the pay-as bid mechanism.

Each Generator can bid a maximum of I blocks of output for each trading period. Bidding in multiple blocks reduces the risk of financial loss. Probability Distribution Function (PDF) is used to predict the bidding prices of rivals, which is based on statistical analysis of historical bidding data. The distribution of the rivals' block bidding prices can be given by normal PDF as:

$$
\operatorname{pdf}\left(\tilde{C}_{i}^{n}\right)=\frac{1}{\sqrt{2 \pi} \zeta_{i}^{n}} \exp \left(-\frac{\left(\tilde{C}_{i}^{n}-\mu_{i}^{n}\right)^{2}}{2 \zeta_{i}^{n}}\right)
$$

Where $\tilde{C}_{i}^{n}$ is the $\mathrm{i}^{\text {th }}$ block price of $\mathrm{n}^{\text {th }}$ rival. $\mu_{i}^{n}, \zeta_{i}^{n}$ are the mean values and standard deviation, respectively, of the $\mathrm{n}^{\text {th }}$ rival of $\mathrm{i}^{\text {th }}$ block.

In this work, bidding strategies are to be developed for one period (one hour) auction only and hence Inter-temporal operating constraints for a generator, such as minimum up and down times and the maximum number of start-up and shutdowns allowed, have not been taken into account.

The profit of $\mathrm{j}^{\text {th }}$ Generator at the considered hour is given by:

$$
\Psi_{j}=\sum_{i=1}^{I} C_{j i} P_{j i}-T C_{j}
$$


Here $\psi_{j}$ and $\mathrm{TC}_{\mathrm{j}}$ are the profit and total production cost for $\mathrm{j}^{\text {th }}$ Generator, respectively. $\mathrm{i}$ is the number of bid blocks for $\mathrm{j}^{\text {th }}$ Generator. $\mathrm{C}_{\mathrm{ji}}$ and $\mathrm{P}_{\mathrm{ji}}$ are the price and quantity offered at block $\mathrm{i}$ for $\mathrm{j}^{\text {th }}$ Generator, respectively and the product of these two gives market revenue. Total production cost can be calculated at a considered hour from (3) as

$$
T C_{j}=A_{j}+B_{j} P_{j}+C_{j} P_{j}^{2}
$$

Here $A_{j}, B_{j}$ and $C_{j}$ are the generator cost coefficients.

$\mathrm{P}_{\mathrm{j}}$ is the total quantity produced by $\mathrm{j}^{\text {th }}$ Generator at a considered time, where

$$
P_{j}=\sum_{i=1}^{I} P_{j i}
$$

Each Generator is presumed to be aware of its rivals' attempt to maximize their profits. In the proposed bidding strategy formulation approach, a Generator maximizes its own profit along with rivals' profits. Thus, this profit maximization problem is multi-objective. Instead of different maximization objectives, a single objective function is formulated from a combination of objectives to be optimized by associating appropriate weights to represent their significance.

$$
\operatorname{Max}\left(\beta_{0}\left(\sum_{j=1}^{N+1} \psi_{j}\right)+\sum_{j=1}^{N+1} \beta_{j} \psi_{j}\right.
$$

where $\sum_{j=0}^{N+1} \beta_{j}=1$.

Here $\beta_{0}$ is the weight associated with the sum of profits and $\beta_{j}$ is the weight representing the importance of individual Generator’s profit.

Subject to constraints

(i) Operating constraints:

- Generation limits

$$
P_{j}^{\min } \leq P_{j} \leq P_{j}^{\max }
$$

- Power demand constraint

$$
P_{D}=\sum_{j=1}^{N+1} P_{j}
$$

(ii) Regulatory Constraint:

- Limitation on bid price

$$
C_{j i}<\text { Price cap }
$$

- Limitation on block bid quantity

$$
P_{j i}{ }^{\min } \leq P_{j i} \leq P_{j i}{ }^{\max }
$$

The optimization problem defined in (5)-(9) can be solved for the optimal block bid price of the $i^{\text {th }}$ block of the $\mathrm{j}^{\text {th }}$ Generator represented by $C_{j i}$.

Using the PDF described in (1), rivals' bidding price can be determined using historical bidding data. Formulating the optimal bidding strategy for Generator A, with objective function (5) and constraints (6)-(9), becomes a stochastic optimization problem, to be solved by GWO algorithm.

\section{PROPOSED SOLUTION ALGORITHM}

The optimization problem formulated in the previous section has been solved by using Monte Carlo (MC) simulation, a technique which obtains a probabilistic approximation of a mathematical problem by using statistical sampling. It performs stochastic simulation using random numbers, and repeatedly calculates the equation to arrive at a solution. These MC simulations are incorporated with GWO, a modern heuristic algorithm based on the behavior of Grey Wolf, to obtain the optimal bidding strategy for a Genco. 


\section{A. MONTE CARLO APPROACH}

- Generate a large number of random samples of block bid prices for all the rivals, considering their PDFs.

- Obtain large trial outcomes, by solving the optimization problem with sample values of block bid prices for all the rivals.

- Calculate the expectation value, taking average of all the trial outcomes.

- The detailed algorithm for solving the optimal bidding problem for Genco A, with N rivals, is given below.

1. Specify the maximum number of MC simulations, $\mathrm{M}$.

2. Initialize the simulation counter, $m=0$.

3. Generate random sample values of bid prices of rivals $\tilde{C}_{i}^{n}(\mathrm{n}=1,2, \ldots, \mathrm{N}$ and $\mathrm{i}=1,2, \ldots, \mathrm{I})$ based on their PDF, as given in (1) under price cap constraints.

4. Obtain mean and standard deviation for each block of Genco A, from bid prices of rivals. It is assumed that Genco A decides its bid prices considering rivals’ bidding behavior.

5. Generate bid prices $C_{j i}$ (with price cap constraints) using normal PDF for each block of Genco A.

6. Use GWO to search the optimal dispatch quantity $\left(P_{j i}\right)$ for each block of all the Gencos that maximizes (5) and store those corresponding prices and quantities as $C_{j i}$ and $P_{j i}$, respectively.

7. Update iteration counter $\mathrm{m}=\mathrm{m}+1$.

8. If $\mathrm{m}<\mathrm{M}$, then go to step 3; else go to step 9 .

9. Calculate the expected value of the optimal bid price, i.e. the average of $C_{j i}^{m}$ and optimal bid quantity, i.e. the average of $P_{j i}^{m}$. Maximized profit for each Genco is the profit, corresponding to these average price and quantity of each block.

\section{B. GREY WOLF OPTIMIZATION}

A new population based GWO algorithm is discussed in this section. The algorithm is based on the leadership hierarchy of grey wolves in nature. Grey wolves follow the leadership hierarchy, as shown in Fig. 1 according to which each wolf is assigned a particular task to perform.

Alpha wolf is at the top of hierarchy and instructions passed from alpha are followed by other wolves according to their position on hierarchy. Beta wolves come second in the hierarchy after alpha, follows the orders given by alpha and they help alpha in searching and hunting the prey. Beta wolves dominate delta and omega wolves. They work as an advisor to alpha and become the leader when alpha dies. Delta wolves assist alpha and beta wolves in hunting and maintaining the discipline among omega wolves. Omega wolves come at last in hierarchy and they aren't much help to other wolves as they do not participate in hunting procedure necessarily. They are allowed to eat after other wolves. The group size is 5-12 on average.

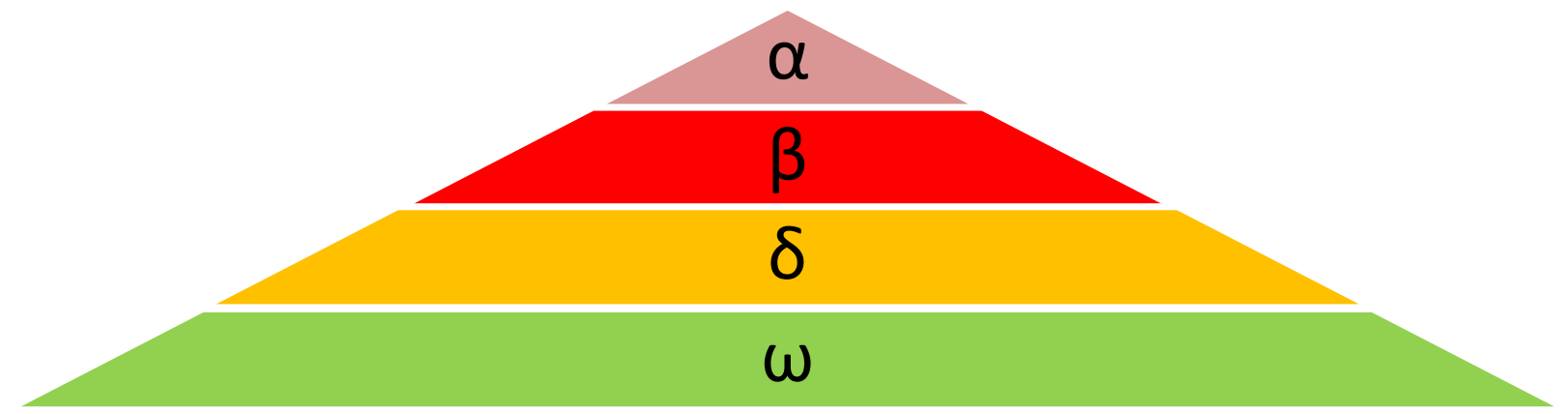

Fig. 1. Hierarchy of Grey Wolf

The important features of hunting mechanism of grey wolves are as follows:

1. Tracking, chasing, and approaching the prey

2. Pursuing, encircling, and harassing the prey until it stops moving

3. Attack towards the prey 
The mathematical modeling of behavior of grey wolves can be done by considering the fittest solution as the alpha $(\alpha)$, second best as the beta $(\beta)$, third best as the delta $(\delta)$ and rest of the candidate solutions are assumed to be omega ( $\omega$ ). Only $\alpha, \beta$ and $\delta$ participate in hunting. The $\omega$ wolves only follow these three wolves.

Encircling the prey is a significant task in hunting procedure, the following equations represents the encircling behavior of grey wolves:

$$
\begin{gathered}
\vec{D}=\left|\vec{C} \cdot \vec{X}_{p}(t)-\vec{X}(t)\right| \\
\vec{X}(t+1)=\vec{X}_{p}(t)-\vec{A} \cdot \vec{D}
\end{gathered}
$$

Where t indicates the current iteration, $\vec{A}$ and $\vec{C}$ are coefficient vectors, $\vec{X}_{p}$ is the position vector of the prey and $\vec{X}$ indicates the position of a grey wolf.

The vectors $\vec{A}$ and $\vec{C}$ are coefficient vectors, $\vec{X}_{p}$ is the position vector of the prey and $\vec{X}$ indicates the position vector of grey wolf.

The vectors $\vec{A}$ and $\vec{C}$ are calculated as follows:

$$
\left.\begin{array}{l}
\vec{A}=2 \vec{a} \cdot \vec{r}_{1}-\vec{a} \\
\vec{C}=2 . \vec{r}_{2}
\end{array}\right\}
$$

Where components of $\vec{a}$ are linearly decreased from 2 to 0 over the course of iterations and $r_{1}, r_{2}$ are random vectors in $[0,1]$.

Grey wolves have the ability to recognize the location of prey and encircle them. The hunt is usually guided by the alpha. The beta and delta might also participate in hunting occasionally. However, in an abstract search space there is no idea about the location of the optimum (prey). In order to mathematically simulate the hunting behavior of grey wolves, suppose that the alpha (best candidate solution) beta, and delta have better knowledge about the potential location of prey. Therefore, the first three best solutions obtained so far are saved and oblige the other search agents (including the omegas) to update their positions according to the position of the best search agent. The following formulas are proposed in this regard.

$$
\begin{gathered}
\vec{D}_{\alpha}=\left|\vec{C}_{1} \cdot \vec{X}_{\alpha}-\vec{X}\right|, \vec{D}_{\beta}=\left|\vec{C}_{2} \cdot \vec{X}_{\beta}-\vec{X}\right|, \vec{D}_{\delta}=\left|\vec{C}_{3} \cdot \vec{X}_{\delta}-\vec{X}\right| \\
\vec{X}_{1}=\vec{X}_{\alpha}-\vec{A}_{1} \cdot\left(\vec{D}_{\alpha}\right), \vec{X}_{2}=\vec{X}_{\beta}-\vec{A}_{2} \cdot\left(\vec{D}_{\beta}\right), \vec{X}_{3}=\vec{X}_{\delta}-\vec{A}_{3} \cdot\left(\vec{D}_{\delta}\right) \\
\vec{X}(t+1)=\frac{\vec{X}_{1}+\vec{X}_{2}+\vec{X}_{3}}{3}
\end{gathered}
$$

Recently GWO has been applied in many fields namely AGC controller design [24], robust generation control strategy [25], computational simulations [26], combined economic load dispatch problem [27], surface wave estimation [28] and training of multi-layer perceptrons [29]. The application of GWO to formulate strategic bidding is explored in this paper. Following section presents simulation results on 4 generators test system.

\section{RESULTS}

This section presents the simulation results of proposed Monte Carlo and GWO approach. A computer program has been developed using MATLAB 2013 and run on a Pentium IV CPU, 2.69 GHz, and 1.84 GB RAM computer. The objective of the optimization routine is to maximize the profit of a specific Genco. A 4 generator system has been considered for validating this simulation approach. The data for the generating units have been taken from [22]. The optimization routine has been addressed by two different load levels. The application of Monte Carlo approach is for forecasting the bid price for participating Gencos. Different bid price predicted for all four Gencos by Monte Carlo simulations are given in Fig. 2. 


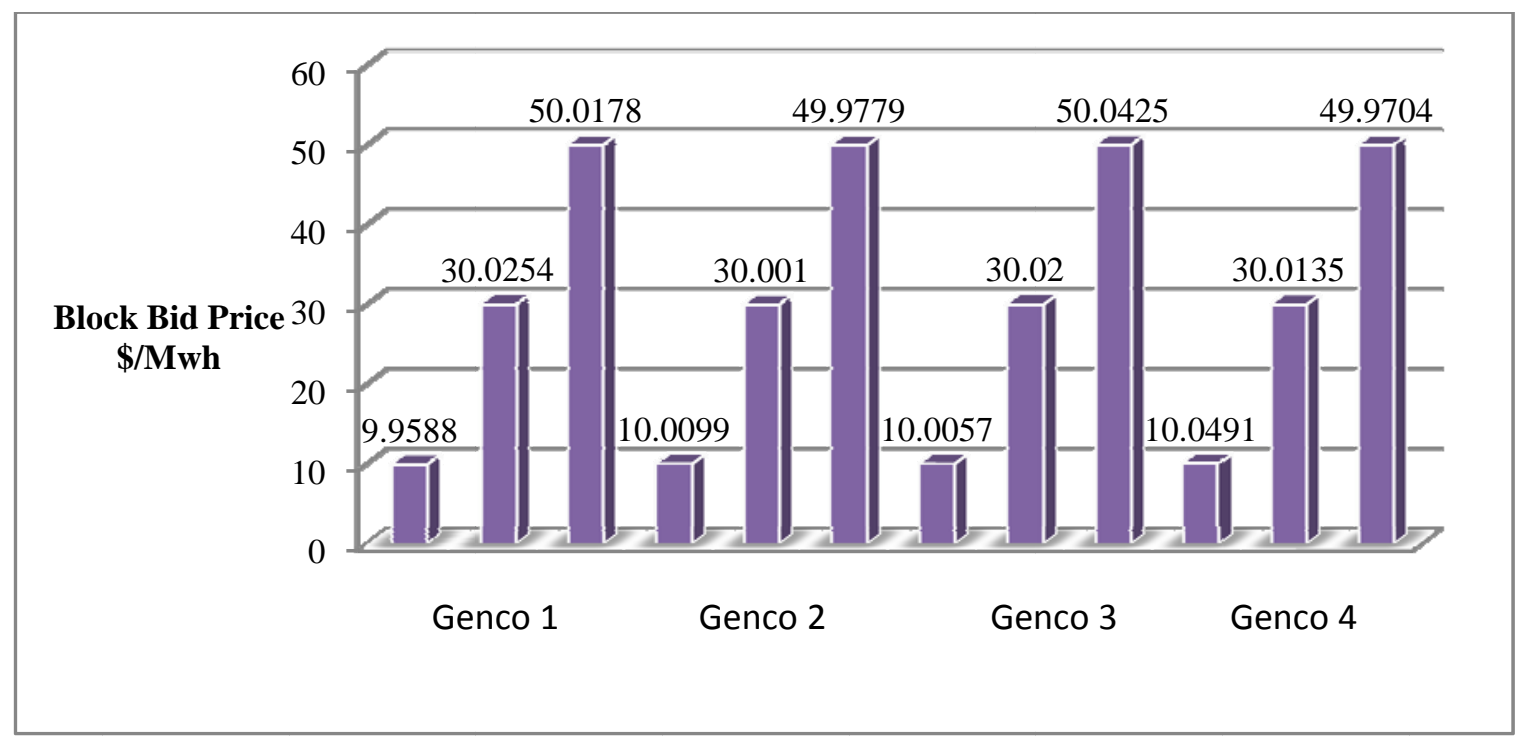

Fig. 2. Bid prices for participating Gencos

On the basis of these bid prices, optimization routine has been run with the aim of profit maximization of Genco 1. The power dispatch results obtained from three different algorithms (GA, PSO and BBO) along with GWO for 1500 system demand is shown in Table 1. It is observed that for Genco 1 (all blocks) the dispatch obtained by GWO are more as compared with all above mentioned three algorithms. The same is observed in the case of Genco 2. However, for Genco 3 and 4 the dispatch is irregular and doesn't follow the same pattern. This suggests that the profit made by company 1 and 2 are more than the others.

\section{Case 1. Dispatch is considered for 1500MW demand.}

Table 1 shows dispatch calculated by different algorithms. Table 2 shows the profit made by the Gencos. It is observed from the Table 2 that the optimization routine successfully computed the dispatch for maximization of the profit of Genco 1. The maximum profit is recorded by GWO based optimization routine. The profit booked by Genco 1 through GA enabled dispatch problem is 9843.609 \$ which are highest amongst all Genco. It is also observed that when optimization routine has been solved by BBO algorithm the dispatch obtained makes the profit of Genco 1 highest. However, significant amount of increment in profit is observed when the dispatch problem is solved through GWO.

Table 1. Dispatch Calculated by different algorithms

\begin{tabular}{|c|c|c|c|c|c|c|c|c|c|c|c|c|}
\hline \multirow{2}{*}{$\begin{array}{c}\text { Dispatch } \\
\text { algorithm } \\
\text { /Genco }\end{array}$} & \multicolumn{3}{|c|}{ Genco 1} & \multicolumn{3}{|c|}{ Genco 2} & \multicolumn{3}{|c|}{ Genco 3} & \multicolumn{3}{|c|}{ Genco4 } \\
\hline & $\begin{array}{c}\text { Bloc } \\
\text { k1 }\end{array}$ & $\begin{array}{c}\text { Bloc } \\
\text { k2 }\end{array}$ & $\begin{array}{c}\text { Bloc } \\
\text { k3 }\end{array}$ & $\begin{array}{c}\text { Bloc } \\
\text { k1 }\end{array}$ & $\begin{array}{c}\text { Bloc } \\
\text { k2 }\end{array}$ & $\begin{array}{c}\text { Bloc } \\
\text { k3 }\end{array}$ & $\begin{array}{c}\text { Bloc } \\
\text { k1 }\end{array}$ & $\begin{array}{c}\text { Bloc } \\
\text { k2 }\end{array}$ & $\begin{array}{c}\text { Bloc } \\
\text { k3 }\end{array}$ & $\begin{array}{c}\text { Bloc } \\
\text { k1 }\end{array}$ & $\begin{array}{c}\text { Bloc } \\
\text { k2 }\end{array}$ & $\begin{array}{c}\text { Bloc } \\
\text { k3 }\end{array}$ \\
\hline $\begin{array}{c}\text { GA } \\
\text { (MW) }\end{array}$ & $\begin{array}{c}102.3 \\
231\end{array}$ & $\begin{array}{c}131.9 \\
642\end{array}$ & $\begin{array}{c}144.6 \\
637\end{array}$ & $\begin{array}{c}104.2 \\
71\end{array}$ & $\begin{array}{c}129.6 \\
656\end{array}$ & $\begin{array}{c}141.1 \\
299\end{array}$ & $\begin{array}{c}102.9 \\
752\end{array}$ & $\begin{array}{c}130.4 \\
406\end{array}$ & $\begin{array}{c}139.9 \\
518\end{array}$ & $\begin{array}{c}103.2 \\
864\end{array}$ & $\begin{array}{c}129.4 \\
298\end{array}$ & $\begin{array}{c}139.8 \\
991\end{array}$ \\
\hline $\begin{array}{l}\text { PSO } \\
\text { (MW) }\end{array}$ & $\begin{array}{c}100.7 \\
686\end{array}$ & $\begin{array}{c}128.5 \\
571\end{array}$ & $\begin{array}{c}150.2 \\
417\end{array}$ & $\begin{array}{c}102.9 \\
785\end{array}$ & $\begin{array}{c}123.8 \\
611\end{array}$ & $\begin{array}{c}146.1 \\
022\end{array}$ & $\begin{array}{c}101.9 \\
354\end{array}$ & $\begin{array}{c}122.9 \\
692\end{array}$ & $\begin{array}{c}145.2 \\
727\end{array}$ & $\begin{array}{c}102.4 \\
722\end{array}$ & $\begin{array}{c}125.4 \\
121\end{array}$ & $\begin{array}{c}148.2 \\
496\end{array}$ \\
\hline $\begin{array}{c}\text { BBO } \\
\text { (MW) }\end{array}$ & $\begin{array}{c}0.101 \\
6\end{array}$ & $\begin{array}{c}193.1 \\
89\end{array}$ & 200 & $\begin{array}{c}0.030 \\
4\end{array}$ & $\begin{array}{c}168.5 \\
841\end{array}$ & $\begin{array}{c}199.9 \\
999\end{array}$ & $\begin{array}{c}0.027 \\
6\end{array}$ & $\begin{array}{c}168.9 \\
699\end{array}$ & 2 & 0.026 & $\begin{array}{c}169.0 \\
716\end{array}$ & $\begin{array}{c}199.9 \\
999\end{array}$ \\
\hline $\begin{array}{l}\text { GWO } \\
\text { (MW) }\end{array}$ & $\begin{array}{c}48.88 \\
203\end{array}$ & $\begin{array}{c}198.5 \\
577\end{array}$ & 200 & $\begin{array}{c}52.11 \\
602\end{array}$ & $\begin{array}{c}193.3 \\
109\end{array}$ & 200 & $\begin{array}{c}11.73 \\
943\end{array}$ & $\begin{array}{c}121.4 \\
915\end{array}$ & 200 & $\begin{array}{c}10.38 \\
665\end{array}$ & $\begin{array}{c}198.7 \\
89\end{array}$ & $\begin{array}{c}65.71 \\
706\end{array}$ \\
\hline $\begin{array}{c}\text { Price } \\
\text { (\$/MWh) }\end{array}$ & $\begin{array}{l}9.958 \\
8\end{array}$ & $\begin{array}{c}30.02 \\
54\end{array}$ & $\begin{array}{c}50.01 \\
78\end{array}$ & $\begin{array}{c}10.00 \\
99\end{array}$ & $\begin{array}{c}30.00 \\
1\end{array}$ & $\begin{array}{c}49.97 \\
79\end{array}$ & $\begin{array}{c}10.00 \\
57\end{array}$ & 30.02 & $\begin{array}{c}50.04 \\
25\end{array}$ & $\begin{array}{c}10.04 \\
91\end{array}$ & $\begin{array}{c}30.01 \\
35\end{array}$ & $\begin{array}{c}49.97 \\
04\end{array}$ \\
\hline
\end{tabular}


Table 2. Profit calculated by different optimization routine

\begin{tabular}{|c|c|c|c|c|}
\hline Algorithm/ Profit in \$ & Genco1 & Genco2 & Genco3 & Genco4 \\
\hline GA & 9843.609 & 9657.957 & 9627.1 & 9605.031 \\
\hline PSO & 9998.332 & 9741.634 & 9691.616 & 9857.289 \\
\hline BBO & 13278.44 & 12787.73 & 12811.46 & 12797.95 \\
\hline GWO & 13304.99 & 13217.28 & 11852.61 & 7959.277 \\
\hline
\end{tabular}

\section{Case 2. Dispatch is considered for $1600 \mathrm{MW}$ demand.}

Table 3 shows dispatch calculated by the optimization routines of GA, PSO, BBO and GWO. Table 4 shows the profit calculated by corresponding Gencos. It is observed that the profit earned by the Genco 1 is higher as compared to other Gencos. This result shows that the objective of optimization routine has been achieved. The comparative analysis of the profit calculated by different algorithms shows that GWO outperforms over rest of the algorithms.

Table 3. Dispatch suggested by different algorithms

\begin{tabular}{|c|c|c|c|c|c|c|c|c|c|c|c|c|}
\hline \multirow{2}{*}{$\begin{array}{c}\text { Dispatch } \\
\text { algorith } \\
\text { m/Genco } \\
\end{array}$} & \multicolumn{3}{|c|}{ Genco 1} & \multicolumn{3}{|c|}{ Genco 2} & \multicolumn{3}{|c|}{ Genco 3} & \multicolumn{3}{|c|}{ Genco4 } \\
\hline & $\begin{array}{c}\text { Bloc } \\
\text { k1 }\end{array}$ & $\begin{array}{c}\text { Bloc } \\
\text { k2 }\end{array}$ & $\begin{array}{c}\text { Block } \\
3\end{array}$ & $\begin{array}{c}\text { Bloc } \\
\text { k1 }\end{array}$ & $\begin{array}{c}\text { Bloc } \\
\text { k2 }\end{array}$ & $\begin{array}{c}\text { Bloc } \\
\text { k3 }\end{array}$ & $\begin{array}{c}\text { Bloc } \\
\text { k1 }\end{array}$ & $\begin{array}{c}\text { Bloc } \\
\text { k2 }\end{array}$ & $\begin{array}{c}\text { Bloc } \\
\text { k3 }\end{array}$ & $\begin{array}{c}\text { Bloc } \\
\text { k1 }\end{array}$ & $\begin{array}{c}\text { Bloc } \\
\text { k2 }\end{array}$ & $\begin{array}{c}\text { Bloc } \\
\text { k3 }\end{array}$ \\
\hline $\begin{array}{c}\text { GA } \\
\text { (MW) }\end{array}$ & $\begin{array}{c}146.2 \\
084\end{array}$ & $\begin{array}{c}144.7 \\
623\end{array}$ & $\begin{array}{c}146.5 \\
239\end{array}$ & $\begin{array}{c}150.7 \\
938\end{array}$ & $\begin{array}{c}150.2 \\
55\end{array}$ & $\begin{array}{c}143.6 \\
37\end{array}$ & $\begin{array}{c}145.5 \\
708\end{array}$ & $\begin{array}{c}133.9 \\
304\end{array}$ & $\begin{array}{c}145.7 \\
721\end{array}$ & $\begin{array}{c}146.2 \\
268\end{array}$ & $\begin{array}{c}146.3 \\
173\end{array}$ & $\begin{array}{c}.0012 \\
34\end{array}$ \\
\hline $\begin{array}{l}\text { PSO } \\
\text { (MW) }\end{array}$ & $\begin{array}{c}169.8 \\
799\end{array}$ & $\begin{array}{c}185.6 \\
391\end{array}$ & $\begin{array}{l}133.8 \\
9763\end{array}$ & 200 & $\begin{array}{c}156.2 \\
683\end{array}$ & $\begin{array}{c}40.43 \\
9\end{array}$ & $\begin{array}{c}104.6 \\
383\end{array}$ & $\begin{array}{c}115.4 \\
158\end{array}$ & $\begin{array}{c}57.15 \\
359\end{array}$ & 200 & $\begin{array}{c}175.8 \\
633\end{array}$ & $\begin{array}{c}60.80 \\
421\end{array}$ \\
\hline $\begin{array}{l}\text { BBO } \\
\text { (MW) }\end{array}$ & $\begin{array}{c}50.22 \\
86\end{array}$ & $\begin{array}{c}199.9 \\
7\end{array}$ & 200 & $\begin{array}{c}40.03 \\
95\end{array}$ & $\begin{array}{c}195.9 \\
764\end{array}$ & 200 & $\begin{array}{c}50.46 \\
49\end{array}$ & $\begin{array}{c}198.6 \\
7\end{array}$ & 200 & $\begin{array}{c}49.19 \\
06\end{array}$ & $\begin{array}{c}197.6 \\
3\end{array}$ & 200 \\
\hline $\begin{array}{l}\text { GWO } \\
\text { (MW) }\end{array}$ & $\begin{array}{c}7.026 \\
276\end{array}$ & 200 & 200 & $\begin{array}{c}0.404 \\
458\end{array}$ & 200 & 200 & $\begin{array}{c}12.87 \\
807\end{array}$ & 200 & 200 & 3.955 & 200 & 200 \\
\hline $\begin{array}{c}\text { Price } \\
\text { \$/MWh }\end{array}$ & $\begin{array}{c}9.958 \\
8\end{array}$ & $\begin{array}{c}30.02 \\
54\end{array}$ & $\begin{array}{c}50.01 \\
78\end{array}$ & $\begin{array}{c}10.00 \\
99\end{array}$ & $\begin{array}{c}30.00 \\
1\end{array}$ & $\begin{array}{c}49.97 \\
79\end{array}$ & $\begin{array}{c}10.00 \\
57\end{array}$ & 30.02 & $\begin{array}{c}50.04 \\
25\end{array}$ & $\begin{array}{c}10.04 \\
91\end{array}$ & $\begin{array}{c}30.01 \\
35\end{array}$ & $\begin{array}{c}49.97 \\
04\end{array}$ \\
\hline
\end{tabular}

Table 4. Profit calculated by different optimization routine

\begin{tabular}{|c|c|c|c|c|}
\hline $\begin{array}{c}\text { Algorithm/ } \\
\text { Profit in \$ }\end{array}$ & Genco1 & Genco2 & Genco3 & Genco4 \\
\hline GA & 10103.15 & 10081.9 & 9886.738 & 4313.106 \\
\hline PSO & 10289.42 & 6147.374 & 5952.958 & 7308.036 \\
\hline BBO & 13327 & 13265 & 13310 & 13280 \\
\hline GWO & 13400.77 & 13395.38 & 13397.83 & 13392.84 \\
\hline
\end{tabular}

\section{OPTIMIZATION PERFORMANCE}

To compare the optimization performance of these algorithms, population sizes, no of iterations are kept same for all. In this work these parameters are chosen as 100 and 1000.Stopping criterions of optimization process are chosen on the basis of execution of optimization process till maximum no. of iterations or the values of objective functions in successive run should have the difference 1e-3. Keeping these parameters, optimization process is executed with all four algorithms. It is observed from Fig. 3 that the optimization process handled by GA got trapped in local minima and the value of the calculated profit for Genco 1 is 9843.609 \$. This profit is $35 \%$ less than the profit calculated by GWO. Moreover the execution time of the optimization routine also puts a question mark on this approach. It is also observed that the convergence point of BBO and GWO are quite close but the values obtained from the optimization process of GWO is more. Fig. 4 shows the convergence characteristics of the values of profit obtained from the optimization processes by GA, PSO and BBO. The profits calculated by these algorithms are less than by $32.16 \%, 30.23 \%$ and $0.5 \%$ respectively as compared with GWO. It is observed that GA and PSO algorithm stopped at local minima. However, a marginal difference has been observed in the values of objective functions in GWO and BBO. 


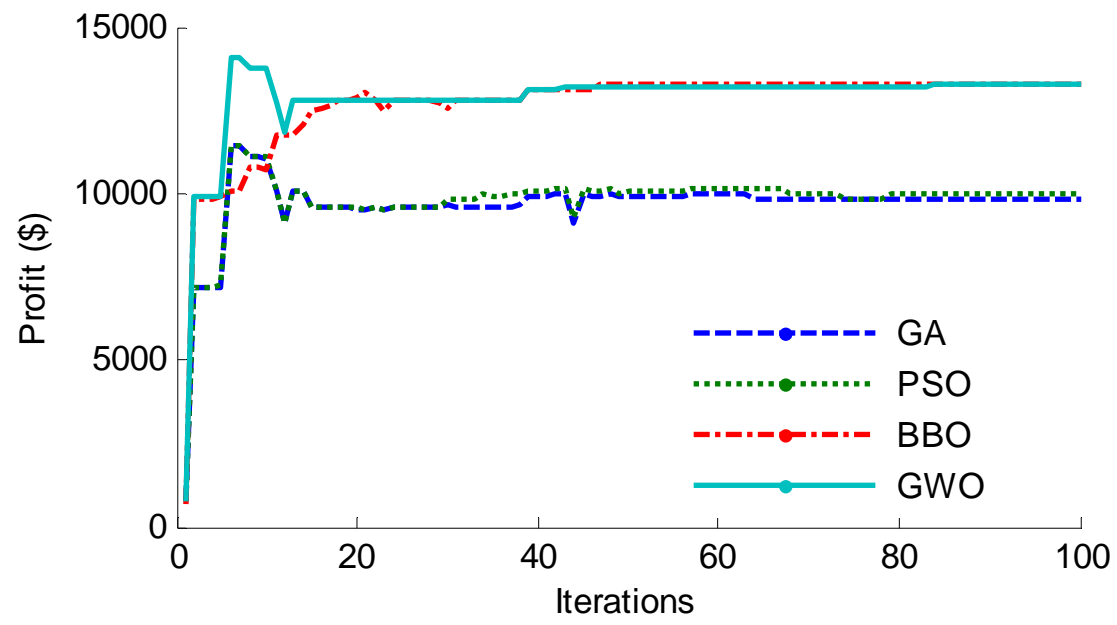

Fig. 3. Convergence curve for $1500 \mathrm{MW}$ demand

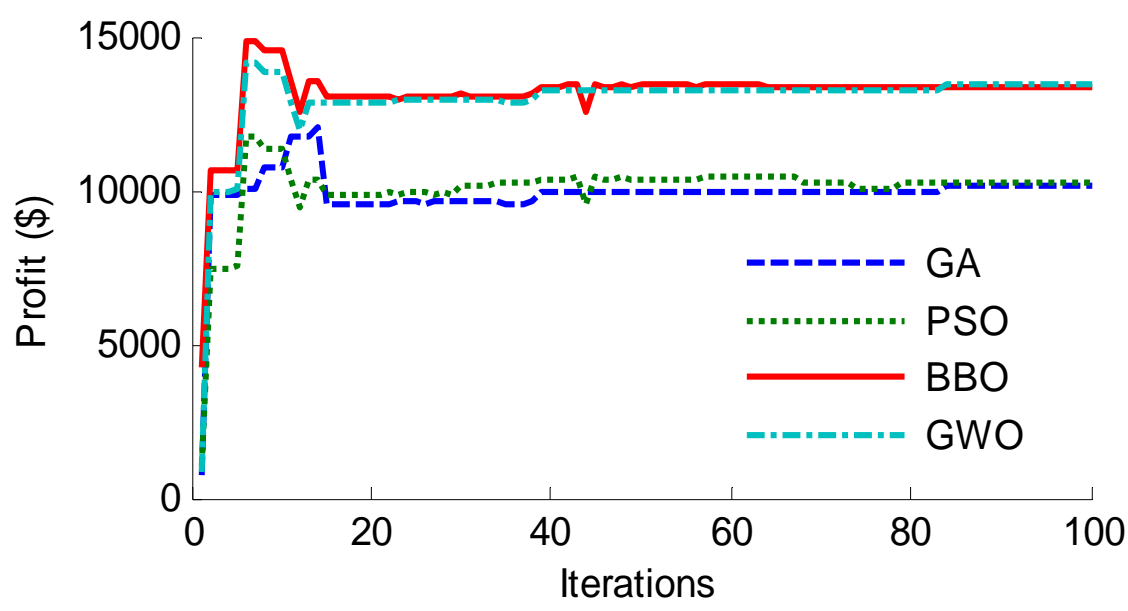

Fig. 4. Convergence curve for 1600 MW demand

\section{CONCLUSION}

In this work, a method based on GWO algorithm is proposed to solve the optimal bidding strategy in an imperfect environment of electricity market under step wise bidding protocol and uniform market clearing price (MCP) environment. A problem which consists of four Gencos is used to verify the feasibility of the proposed method. Rivals behavior is predicted by historical data and normal probability density function and the same is applied to simulate it. The results are found for two different load levels, $1500 \mathrm{MW}$ and $1600 \mathrm{MW}$. The proposed method gives promising results in comparison to other heuristic algorithms (GA, PSO and BBO). In future, an attempt will be incorporated to include inter-temporal operating constraints of generators.

\section{REFERENCES}

[1] Fushuan Wen and A. K. David, "Optimal bidding strategies and modeling of imperfect information among competitive generators," in IEEE Transactions on Power Systems, vol. 16, no. 1, pp. 15-21, Feb 2001.

[2] A. K. David and Fushuan Wen, "Strategic bidding in competitive electricity markets: a literature survey," 2000 Power Engineering Society Summer Meeting, Seattle, WA, 2000, pp. 2168-2173 vol. 4.

[3] A. K. David, "Competitive bidding in electricity supply," in IEE Proceedings C - Generation, Transmission and Distribution, vol. 140, no. 5, pp. 421-426, Sept. 1993.

[4] E. S. Huse, I. Wangensteen and H. H. Faanes, "Thermal power generation scheduling by simulated competition," in IEEE Transactions on Power Systems, vol. 14, no. 2, pp. 472-477, May 1999

[5] Li Ma, Wen Fushuan and A. K. David, "A preliminary study on strategic bidding in electricity markets with step-wise bidding protocol," IEEE/PES Transmission and Distribution Conference and Exhibition, 2002, pp. 1960-1965 vol.3.

[6] H. Song, C. C. Liu, J. Lawarree and R. W. Dahlgren, "Optimal electricity supply bidding by Markov decision process," in IEEE Transactions on Power Systems, vol. 15, no. 2, pp. 618-624, May 2000.

[7] F. S. Wen, A. K. David, Strategic bidding for electricity supply in a day-ahead energy market, Electric Power Systems Research, Volume 59, Issue 3, 31 October 2001, Pages 197-206.

[8] F. S. Wen and A. K. David, "Optimally co-ordinated bidding strategies in energy and ancillary service markets," in IEE Proceedings Generation, Transmission and Distribution, vol. 149, no. 3, pp. 331-338, May 2002.

[9] C. W. Richter, G. B. Sheble and D. Ashlock, "Comprehensive bidding strategies with genetic programming/finite state automata," in IEEE Transactions on Power Systems, vol. 14, no. 4, pp. 1207-1212, Nov 1999. 
[10] P. Bajpai and S. N. Singh, "Fuzzy Adaptive Particle Swarm Optimization for Bidding Strategy in Uniform Price Spot Market," in IEEE Transactions on Power Systems, vol. 22, no. 4, pp. 2152-2160, Nov. 2007.

[11] R. W. Ferrero, S. M. Shahidehpour and V. C. Ramesh, "Transaction analysis in deregulated power systems using game theory," in IEEE Transactions on Power Systems, vol. 12, no. 3, pp. 1340-1347, Aug 1997.

[12] K. Seeley, J. Lawarree and C. C. Liu, "Analysis of electricity market rules and their effects on strategic behavior in a noncongestive grid," in IEEE Transactions on Power Systems, vol. 15, no. 1, pp. 157-162, Feb 2000.

[13] Shangyou Hao, "A study of basic bidding strategy in clearing pricing auctions," in IEEE Transactions on Power Systems, vol. 15, no. 3, pp. 975-980, Aug 2000.

[14] Xiaohong Guan, Yu-Chi Ho and D. L. Pepyne, "Gaming and price spikes in electric power markets," in IEEE Transactions on Power Systems, vol. 16, no. 3, pp. 402-408, Aug 2001.

[15] Wang Xian, Li Yuzeng and Zhang Shaohua, "Oligopolistic equilibrium analysis for electricity markets: a nonlinear complementarity approach," in IEEE Transactions on Power Systems, vol. 19, no. 3, pp. 1348-1355, Aug. 2004.

[16] M. V. Pereira, S. Granville, M. H. C. Fampa, R. Dix and L. A. Barroso, "Strategic bidding under uncertainty: a binary expansion approach," in IEEE Transactions on Power Systems, vol. 20, no. 1, pp. 180-188, Feb. 2005.

[17] R. Herranz, A. Munoz San Roque, J. Villar and F. A. Campos, "Optimal Demand-Side Bidding Strategies in Electricity Spot Markets," in IEEE Transactions on Power Systems, vol. 27, no. 3, pp. 1204-1213, Aug. 2012.

[18] M. Kazemi, B. Mohammadi-Ivatloo and M. Ehsan, "Risk-Constrained Strategic Bidding of GenCos Considering Demand Response," in IEEE Transactions on Power Systems, vol. 30, no. 1, pp. 376-384, Jan. 2015.

[19] S. J. Kazempour, A. J. Conejo and C. Ruiz, "Strategic Bidding for a Large Consumer," in IEEE Transactions on Power Systems, vol. 30, no. 2, pp. 848-856, March 2015.

[20] B. Ansari and A. Rahimi-Kian, "A Dynamic Risk-Constrained Bidding Strategy for Generation Companies Based on Linear Supply Function Model," in IEEE Systems Journal, vol. 9, no. 4, pp. 1463-1474, Dec. 2015.

[21] F. A. Campos, A. Muñoz San Roque, E. F. Sánchez-Úbeda and J. Portela González, "Strategic Bidding in Secondary Reserve Markets," in IEEE Transactions on Power Systems, vol. 31, no. 4, pp. 2847-2856, July 2016.

[22] P. Jain, A. Agarwal, N. Gupta, R. Sharma, U. Paliwal and R. Bhakar, "Profit maximization of a generation company based on Biogeography based Optimization," 2012 IEEE Power and Energy Society General Meeting, San Diego, CA, 2012, pp. 1-6.

[23] Seyedali Mirjalili, Seyed Mohammad Mirjalili, Andrew Lewis, Grey Wolf Optimizer, Advances in Engineering Software, Volume 69, March 2014, Pages 46-61.

[24] E. Gupta, A. Saxena, "Grey wolf optimizer based regulator design for automatic generation control of interconnected power system,” Cogent Engineering, 3(1), 1151612, Feb. 2016.

[25] E. Gupta, A. Saxena, "Robust generation control strategy based on grey wolf optimizer," in Journal of Electrical Systems, vol. 11, no. 2, pp. 174-188, 2015.

[26] C. Muro, R. Escobedo, L. Spector, and R. Coppinger, "Wolf-pack (Canis lupus) hunting strategies emerge from simple rules in computational simulations,” Behavioural processes, vol. 88, pp. 192-197, 2011.

[27] Song HM, Sulaiman MH, Mohamed MR, "An application of Grey Wolf Optimizer for solving combined economic emission dispatch problems,” Int. Rev. Model. Simul., vol. 7, no. 5, pp. 838-844, 2014.

[28] Xianhai Song, Li Tang, Sutao Zhao, Xueqiang Zhang, Lei Li, Jianquan Huang, Wei Cai, "Grey Wolf Optimizer for parameter estimation in surface waves," Soil Dynamics and Earthquake Engineering, vol. 75, Aug 2015.

[29] Mirjalili S., "How effective is the Grey Wolf Optimizer in training multi-layer perceptrons," Appl. Intell., 2015. 